\title{
Clinical and neuroimaging determinants of minimally conscious and persistent vegetative states after acute stroke
}

\author{
Emre Kumral, MD; Fatma Ece Bayam, MD; Bedriye Köken, MD; \\ Can Emre Erdoğan, MD \\ Department of Neurology, Ege University Medical School Hospital, Izmir, Turkey
}

\section{ORIGINAL ARTICLE \\ Journal of Neurocritical Care \\ Revised: June 1, 2019 \\ Accepted: June 3, 2019 \\ Corresponding Author:
Emre Kumral, MD \\ Stroke Unit, Department of Neurology, Ege University Faculty of Medicine, Bornova, Izmir 35100, Turkey \\ Tel: +90-532-2165213 \\ Fax: +90-232-4634626 \\ E-mail:emre.kumral@ege.edu.tr}

Background: Patients with persistent vegetative state (PVS) show no evidence of awareness of self or their environment, and those with minimally conscious state (MCS) have severely impaired consciousness with minimal but definite behavioral evidence of self or environmental awareness after stroke. Neuroimaging and clinical characteristics separating these two close consciousness states after stroke were insufficiently studied.

Methods: We conducted a hospital-based cohort study of all patients with stroke (2011 to 2017) who underwent 3T magnetic resonance imaging and consciousness assessment after 3 months of inclusion. Univariate and multivariate regression analyses were used to estimate the relative risk of neuroimaging markers for differentiation of PVS and MCS.

Results: Of 3,600 eligible subjects, 323 patients (0.09\%) had PVS and 93 (0.02\%) had MCS (mean age, $62.25 \pm 13.4$ years). Higher stroke volume was strongly associated with PVS compared to MCS (odds ratio [OR], 0.99; 95\% confidence interval [Cl], 0.98 to 1.00; $P=0.001)$. On univariate analysis, cingulate gyrus (OR, $2.7 ; 95 \% \mathrm{Cl}, 1.62$ to $4.36 ; P=0.001)$ and corpus callosum $(\mathrm{OR}, 2.1 ; 95 \% \mathrm{Cl}, 1.28$ to $3.44 ; P=0.003$ ) involvement was significantly associated with $P V S$. However, on multivariate analysis, only cingulate gyrus involvement was independently associated with PVS $(\mathrm{OR}, 2.2 ; 95 \% \mathrm{Cl}, 1.33$ to $3.72 ; P=0.002)$.

Conclusion: Our results indicate that PVS and MCS are different consciousness states according to clinical and neuroimaging findings. To predict outcome, cognitive performance of these patients should be well questioned after stroke.

Keywords: Minimally conscious state; Persistent vegetative state; Ischemic stroke; Hemorrhagic stroke; Cognition disorders; Outcome

\section{INTRODUCTION}

Consciousness is the state of full awareness of the self and one's relationship to the environment. Clinically, the level of consciousness of a patient is defined operationally at the bedside by the responses of the patient to the examiner. Consciousness has two major components: arousal and awareness $[1,2]$. The content of consciousness represents the sum of all functions mediated at a cerebral cortical level, including both cognitive and affective responses. Very few surviving patients with severe forebrain damage after stroke remain in an eyes-closed coma for more than 10 to 14 days; then, a vegetative state (also called coma vigil or

(C) 2019 The Korean Neurocritical Care Society

This is an Open Access article distributed under the terms of the Creative Commons Attribution Non-Commercial License (http://creativecommons.org/licenses/by-nc/4.0/) which permits unrestricted noncommercial use, distribution, and reproduction in any medium, provided the original work is properly cited. 
apallic state) usually replaces coma by that time [3]. The American Neurological Association advises that persistent vegetative state (PVS) can be considered only in patients in that state for 1 month [4]. The minimally conscious state (MCS) is a concept that was defined by the Aspen Workgroup [5], which identifies a condition of severely impaired consciousness in which minimal but definite behavioral evidence of self or environmental awareness is demonstrated.

However, the neuroimaging markers of PVS and MCS and their relative contributions after acute stroke have not been precisely defined. A large body of evidence suggests that several brain magnetic resonance imaging (MRI) markers may be linked to the incidence of PVS and MCS: stroke subtype (ischemic or hemorrhagic), stroke volume and gross location (with a detrimental effect of hemispheric stroke), strategic locations, multiple lesions, and recurrent stroke [6-10]. Surprisingly, the contribution of previous cognitive disorders to consciousness disorders has not been confirmed by systematic studies of stroke cohorts despite having been largely emphasized on poststroke cognitive disorders [11]. Most of the abovementioned studies presented some limitations. First, the studies focused on neurocognitive disorders rather than the more frequent consciousness disorders. Second, most studies separately examined the association between PVS and MCS and various determinants without evaluating their relative contributions. Lastly, most studies were based on a mass-effect approach (i.e., global tissue damage), which precluded any conclusions on the contribution of precise stroke locations and patterns (e.g., strategic regions). Hence, we aimed to find neuroimaging determinants of poststroke PVS and MCS based on clinical and neuroimaging findings in a large, prospective cohort of patients with stroke. To improve the determination of clinical-neuroimaging relationship, we used an optimized clinical questionnaire (OCQ) and combined both quantitative measures related to structure loss and qualitative measures related to the presence of strategic lesions.

\section{METHODS}

Between September 2011 and November 2017, 3,600 patients in our comprehensive stroke center had been prospectively included. Details of the stroke registry protocol have been previously reported [12]. All patients were enrolled within 48 hours of stroke onset. Briefly, all consenting Turkish-speaking patients aged between 40 and 80 years who were hospitalized for acute $(<30$ days) cerebral infarct or hemorrhage with initial positive imaging and had a reliable informant and conditions affecting cognition were included. Clinical evaluation was performed in the neurointensive care unit by physicians and nurses. Baseline examinations, including questionnaire administration, blood chemistry, and extracranial and intracranial ultrasonography, were performed in all patients to confirm the referral diagnosis and screen for risk factors. The baseline examinations and definitions of vascular disease and risk factors are described briefly below.

Prospectively recorded variables included age, sex, previous stroke, risk factors, blood pressure and modified Rankin Scale (mRS) score [13], National Institutes of Health Stroke Scale (NIHSS) [14], and Glasgow Coma Scale (GCS) [15] scores at the time of admission, etiological subtypes in patients with ischemic stroke (IS) according to the Trial of ORG 10172 in Acute Stroke Treatment (TOAST) [16] classification, topography of infarcts and hemorrhage on MRI studies, in-hospital recurrent stroke, neurologic and systemic complications, and treatment modalities including osmotherapy, decompression surgery, thrombolysis, and thrombectomy. The study was performed in accordance with institutional guidelines and approved by the Ethics Committee of the Ege University Medical Center Institutional Review Board, and a written informed consent was obtained from all the relatives or parents of the participants. Ege University Medical Ethical Committee was approved this study following the principles outlined in the Helsinki Declaration before starting the study (2010).

\section{Assessment of consciousness state}

Clinical consciousness examination of each patient was performed at stroke onset in the neurointensive care and stroke unit. A consciousness assessment was performed every hour after entry into the neurointensive care and stroke unit. Consciousness state of the patients were determined by two trained neurologists and followed continuously in the neurointensive care unit. The kappa value between the two neurologists who performed the final consciousness assessment was 0.98. Coma was considered if there is a state of unresponsiveness in which the patient lies with eyes closed, cannot be aroused to respond appropriately to stimuli even with vigorous stimulation, and does not have localized responses or discrete defensive movements. PVS denotes the recovery of crude cycling of arousal states heralded by the appearance of "eyes-open" periods in an unresponsive patient. PVS was presumed if the patient remained in that state for at least 30 days according to the criteria of the Multisociety Task Force on PVS $[17,18]$. MCS was considered if the patient had a condition of severely impaired consciousness in which minimal but definite behavioral evidence of self or environmental awareness is demonstrated. The differential diagnosis of PVS and MCS was made according to criteria of the Aspen Working Group [5]. An OCQ of the Aspen Working Group Criteria was administered, and evidence of limited but clearly discernible self or environmental 
awareness was obtained according to one or more of the following behaviors: (1) following simple command; (2) gestural or verbal "yes/no" responses (independent of accuracy); (3) intelligible verbalization; (4) purposeful behavior; and (5) affective behavior.

\section{Lesion location}

MRI was performed within the first week of admission by 3T scanners (Siemens Sonata, Siemens Medical Solutions, Erlangen, Germany). MRI scanners consisted of axial T1- and T2-weighted spinecho, T2-weighted fluid-attenuated inversion recovery (FLAIR), and diffusion-weighted imaging (DWI). Sagittal and axial FLAIR or DWIs were used to assess lesion size and site. Two neuroradiologists who evaluated the images were blinded to the clinical picture (interobserver agreement, 0.97). The presence, type, and number of strategic lesions and volume for consciousness impairment were determined using the 3-dimensional-T1 BRAVO sequence and the STandards for ReportIng Vascular changes on $n E$ Euroimaging (STRIVE) criteria [19]. Senior investigators manually segmented the lesions on native 3D-T1 MRI data sets using MRIcron (a cross-platform NIfTI format image viewer). Normalized lesion masks were used to compute the stroke volume that considered all strokes. Strategic sites include foci within the following regions: left and right middle frontal gyrus, left and right middle and superior temporal gyrus, left and right parietal cortex (angular and supramarginal gyri), putamen, pallidum, cingulate gyrus and corpus callosum, left arcuate fasciculus, anterior and middle parts of the left and right thalami, ventral and dorsal midbrain, and dorsal pons. The white matter hyperintensities (WMHs) burden was assessed using the Fazekas scale score [20] on the basis of FLAIR sequence. Brain microbleeds were defined as small ( $<10 \mathrm{~mm}$ in diameter) areas of signal void according to the brain observer microbleed scale criteria [21]. T2 sequence was used to assess the presence of dilated perivascular spaces.

\section{Stroke mechanisms}

After analyzing DWI, angiographic, and clinical data, stroke mechanisms were categorized according to previous descriptions. Large-artery disease (LAD) was presumed in patients with symptomatic carotid artery stenosis $>50 \%$. Small-artery disease or local branch occlusion was defined in patients with infarcts $<15$ $\mathrm{mm}$ in diameter localized in the deep regions of the brain or in the brainstem without $\mathrm{LAD}$ and cardiac embolism (CE). CE was considered in patients with a source of CE. Strokes of other determined or undetermined causes were also recorded. The presence, type, and volume of cerebral hemorrhage were noted.

\section{Concomitant risk factors}

Among cognitive risk factors, minor or major neurocognitive disorder was recorded. Previous cognitive status of every patient were asked from their practitioner or from the National Health Data System. Vascular risk factors including hypertension, current cigarette smoking, and serum total cholesterol, low-density lipoprotein cholesterol, high-density lipoprotein cholesterol, triglyceride, glucose, and glycated hemoglobin levels in hemolysates were determined. Apolipoprotein E (ApoE) genotype was determined by polymerase chain reaction, and patients were classified by presence or absence of at least 1 ApoE epsilon 4 allele.

\section{Statistical analyses}

Proportions, odds ratios (ORs), and means of baseline, clinical, and neuroimaging characteristics were compared between patients with PVS and those with MCS using the chi-square test, logistic regression, and analysis of variance, respectively. Univariate associations between PVS and MCS according to age, sex, vascular risk factors, previous stroke and recurrence, history of neurocognitive disorder, stroke subtypes, strategic locations, white matter lesions, microbleeds, and dilated perivascular spaces were analyzed using logistic regression to estimate unadjusted ORs. Association between PVS and MCS was determined to estimate models using multivariate logistic regression analysis with backward stepwise selection, which is based on the probability of the likelihood-ratio statistic according to the maximum partial likelihood estimates by including clinical and neuroimaging findings. The selection process of the final model was performed in three steps by entering all variable(s) in binary logistic regression analysis. In the last model of variables, changes in -2 log-likelihood and model $\log$ likelihood and significance of the change were obtained. Probability values $<0.05$ were considered statistically significant. All statistical analyses were performed using SPSS version 18.0 (SPSS Inc., Chicago, IL, USA).

\section{RESULTS}

Data were available for 323 patients with PVS and MCS (mean age, $62.25 \pm 13.4$ years; male, $41 \%$ ). Characteristics of patients with PVS $(\mathrm{n}=230)$ and MCS $(\mathrm{n}=93)$ are presented in Table 1. Of 230 patients with PVS, 83\% had coma state at stroke onset, and the rest showed transition from severe consciousness disturbances to coma, while 70 patients (75\%) with MCS had coma, 15 (16\%) had transition from vegetative state to MCS in 3 months, and eight (9\%) had progressive deterioration to MCS after stroke. Almost all patients had an mRS score $\geq 4$ (median, 5 [4 to 5]), NIHSS score $\geq 12$ (median, 24 [12 to 32]), and GCS score $\leq 7$ 
Table 1. Characteristics of patients with persistent vegetative state and minimally conscious state

\begin{tabular}{|c|c|c|c|}
\hline Characteristic & Patients with PVS $(n=230)$ & Patients with MCS $(n=93)$ & $P$ value \\
\hline \multicolumn{4}{|l|}{ Demographic characteristic } \\
\hline Age (yr) & $63 \pm 13$ & $62 \pm 14$ & 0.540 \\
\hline Male sex & $123(54)$ & $44(47)$ & 0.120 \\
\hline \multicolumn{4}{|l|}{ Vascular risk factor } \\
\hline Previous stroke & $30(13)$ & $16(17)$ & 0.210 \\
\hline History of MND & $41(18)$ & $9(10)$ & 0.090 \\
\hline Hypertension & $116(50)$ & $46(49)$ & 0.490 \\
\hline Diabetes mellitus & $34(15)$ & $16(17)$ & 0.350 \\
\hline Cigarette smoking & $47(20)$ & $21(23)$ & 0.390 \\
\hline Atrial fibrillation & $51(22)$ & $18(19)$ & 0.330 \\
\hline Hyperlipidemia & $110(48)$ & $47(51)$ & 0.380 \\
\hline ApoE $\varepsilon 4$ allele & $45(20)$ & $26(28)$ & 0.070 \\
\hline WMH burden (Fazekas scale 1-3) & $128(56)$ & $43(46)$ & 0.080 \\
\hline Microbleeds & $23(10)$ & $6(7)$ & 0.220 \\
\hline Stroke volume $\left(\mathrm{cm}^{3}\right)$ & $165 \pm 57$ & $136 \pm 59$ & 0.001 \\
\hline Presence of dilated perivascular spaces & $45(20)$ & $7(8)$ & 0.004 \\
\hline \multicolumn{4}{|l|}{ Stroke pathogenesis } \\
\hline Large-artery disease & $95(41)$ & $42(45)$ & 0.300 \\
\hline Cardioembolic stroke & $54(24)$ & $21(23)$ & 0.490 \\
\hline Small-artery disease & $13(6)$ & $3(3)$ & 0.270 \\
\hline Other causes & $41(38)$ & $17(19)$ & 0.520 \\
\hline Unknown cause & $5(2)$ & $2(2)$ & 0.680 \\
\hline Intracerebral hemorrhage & $30(13)$ & $8(9)$ & 0.180 \\
\hline \multicolumn{4}{|l|}{ Clinical characteristics at admission } \\
\hline NIHSS at stroke onset & $24(10-32)$ & $24(17-29)$ & \\
\hline Modified Rankin Scale score at stroke onset & $5(4-5)$ & $5(4-5)$ & \\
\hline Glasgow coma score (eye-opening, motor, and verbal) & $5(3-7)$ & $6(3-7)$ & \\
\hline One pupil fixed and dilated & $87(38)$ & $25(27)$ & 0.070 \\
\hline \multicolumn{4}{|l|}{ Treatment } \\
\hline Thrombolysis & $78(34)$ & $35(38)$ & 0.310 \\
\hline Thrombectomy & $16(7)$ & $5(5)$ & 0.400 \\
\hline Decompression surgery & $57(25)$ & $21(23)$ & 0.390 \\
\hline Osmotherapy & $158(69)$ & $71(76)$ & 0.110 \\
\hline Mechanical ventilation & $110(48)$ & $36(39)$ & 0.090 \\
\hline
\end{tabular}

Values are presented as mean \pm SD, number $(\%)$, or median (interquartile range).

PVS, persistent vegetative state; MCS, minimally conscious state; MND, major or minor neurocognitive disorder; ApoE, apolipoprotein $E_{\text {; }}$ WMH, white matter hyperintensity; NIHSS, National Institutes of Health Stroke Scale.

(median, $5[3$ to 7$]$ ) at stroke onset. Most patients $(\mathrm{n}=285,88 \%)$ presented with IS, 38 (12\%) had hemorrhage, and 24 (7.4\%) had recurrent stroke between inclusion and 3-month follow-up. Fifty patients (15.5\%) (28 [9\%] with minor and 22 [7\%] with major neurocognitive disorder) had prestroke neurocognitive disorders, but there was no statistically significant difference between the two groups.

Univariate analysis of lesion location showed that the cingulate gyrus $(\mathrm{OR}, 2.14 ; 95 \%$ confidence interval [CI], 1.30 to
3.51; $P=0.003)$ and corpus callosum (OR, 1.68; 95\% CI, 1.30 to 2.75 ; $P=0.004)$ were more frequently involved in patients with PVS. There were more patients with multiple lesions in those with PVS (102 [44\%]) compared with those with MCS (32 [34\%]), but it did not reach a significant level in the analysis. Isolated thalamic and midbrain lesions were found in two patients with MCS, while others had additional supratentorial lesions (Table 2). On the standardized OCQ, the factors related strongly to MCS including following simple command, gestural 
Table 2. Clinical and lesion characteristics of patients with minimally conscious state and persistent vegetative state at 3 months poststroke

\begin{tabular}{|c|c|c|c|}
\hline Characteristic & Patients with PVS $(n=230)$ & Patients with MCS $(n=93)$ & $P$ value \\
\hline \multicolumn{4}{|l|}{ Optimized clinical questionnaire } \\
\hline Following simple command & $1(0.4)$ & $25(27)$ & 0.001 \\
\hline Gestural or verbal "yes/no" response & 0 & $29(31)$ & 0.001 \\
\hline Intelligible verbalization & 0 & $34(37)$ & 0.001 \\
\hline \multicolumn{4}{|l|}{ Purposeful behavior } \\
\hline Response to linguistic content of questions & 0 & $38(41)$ & 0.001 \\
\hline Touching or holding objects & $1(0.4)$ & $55(59)$ & 0.001 \\
\hline Sustained visual fixation or tracking & $3(1)$ & $52(56)$ & 0.001 \\
\hline Reaching for objects in appropriate direction & $2(0.9)$ & $72(77)$ & 0.001 \\
\hline \multicolumn{4}{|l|}{ Affective behavior } \\
\hline Appropriate smiling or crying & $9(4)$ & $59(63)$ & 0.001 \\
\hline \multicolumn{4}{|l|}{ Lesion location and types } \\
\hline \multicolumn{4}{|l|}{ Strategic regions } \\
\hline Frontal & $163(71)$ & $59(63)$ & 0.140 \\
\hline Parietal & $173(75)$ & $75(81)$ & 0.190 \\
\hline Temporal & $161(70)$ & $57(61)$ & 0.080 \\
\hline Putamen and pallidum & $114(50)$ & $40(43)$ & 0.170 \\
\hline Cingulate gyrus & $162(70)$ & $44(47)$ & 0.001 \\
\hline Corpus callosum & $161(65)$ & $49(53)$ & 0.004 \\
\hline Arcuate fasciculus & $84(37)$ & $37(40)$ & 0.340 \\
\hline Thalamus & $34(15)$ & $19(20)$ & 0.140 \\
\hline Midbrain & $26(11)$ & $15(26)$ & 0.160 \\
\hline Cerebellum & $22(10)$ & $9(10)$ & 0.560 \\
\hline Multiple lesions & $102(44)$ & $32(34)$ & 0.060 \\
\hline Bilateral hemispheric lesions & $46(20)$ & $15(16)$ & 0.260 \\
\hline Supra- and infratentorial lesions & $52(23)$ & $21(23)$ & 0.560 \\
\hline
\end{tabular}

Values are presented as number (\%).

PVS, persistent vegetative state; MCS, minimally conscious state.

or verbal "yes/no" response, intelligible verbalization, and purposeful and affective behaviors $(P=0.001$, respectively). Few patients with PVS $(<1 \%)$ showed limited visual fixation or tracking and inappropriate attempt to reach objects.

In the multiple stepwise linear regression analysis, three factors that were independently associated with the PVS compared to MCS were selected: cingulate gyrus involvement, stroke volume, and presence of dilated perivascular spaces (Table 3). Among the strategic regions, cingulate gyrus damage was strongly related to PVS compared to MCS (OR, 2.2; 95\% CI, 1.33 to 3.72; $P=0.002$ ). Assessments of a possible threshold effect of WMH burden, number of microbleeds, stroke recurrence, multiple infarcts, and treatment strategies did not affect the main findings, while there was an independent effect of presence of dilated perivascular spaces on consciousness state (OR, 3.2; 95\% CI, 1.34 to 7.68 ; $P=0.004$ ). Stroke volume also played an important role, which accounted for severe loss of consciousness in patients with PVS compared to
$\operatorname{MCS}(\mathrm{OR}, 0.99 ; 95 \% \mathrm{CI}, 0.98$ to $1.00 ; P=0.001)$.

\section{DISCUSSION}

The major finding of this prospective, hospital-based study of poststroke patients is that, of the different MRI markers, (1) stroke volume and (2) the presence of a lesion within strategic regions (cingulate gyrus and corpus callosum) were the main determinants of PVS compared with MCS. Differentiating the PVS from MCS is often one of the most challenging tasks in the stroke unit. These two clinical entities are close consciousness states, but there is a critical level of awareness manifested by purposeful and affective behaviors that differentiates both clinical pictures [22]. For the explanation of this critical level, we observed that lesion volume and some regional involvement are critical. Most studies measuring volumetric indices in PVS/MCS were based on traumatic [23] or anoxic brain injury [24], while 
Table 3. Univariate and multivariate relationship between minimally conscious state and persistent vegetative state according to neuroimaging markers in the study population at 3 months poststroke

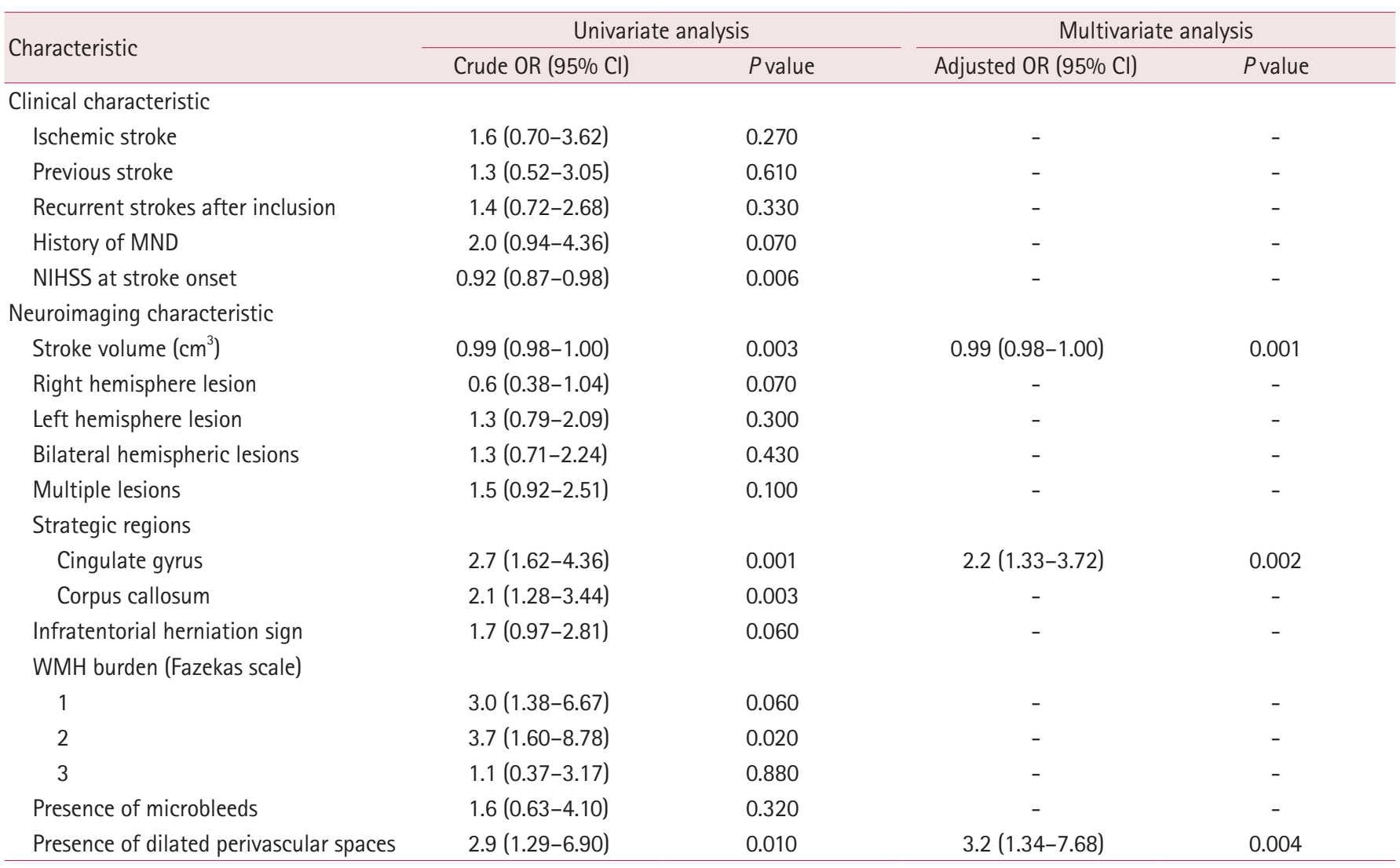

$\mathrm{OR}$, odds ratio; $\mathrm{Cl}$, confidence interval; $\mathrm{MND}$, major or minor neurocognitive disorder; NIHSS, National Institutes of Health Stroke Scale; WMH, white matter hyperintensity.

poststroke pathologic studies showed severe tissue damage with diffuse axonal loss in patients with PVS and only focal brain damage in some patients with MCS [25]. Our and previous findings suggest that significant variations may exist in the underlying mechanisms of cognitive disabilities and residual brain function accompanying MCS.

There are no clear neuroimaging criteria in determining when PVS becomes permanent and MCS appears after severe stroke. One reason for the inability to predict permanence early in the course of PVS is that patients usually have severely damaged cerebral hemispheres combined with a relatively intact brainstem [26]. Our patients with PVS presented likely similar pattern of regional involvement, recurrent stroke, multiple lesions, presence and extent of WMH, and microbleeds compared with those with MCS. The common denominator of PVS may be damage to the corticostriato-pallidal-thalamocortical loops that are critical for the function of the frontal lobes [27]. As we noted in our patients with PVS and MCS, severe damage affected the ventral striatum, ventral pallidum, and mediodorsal nucleus of the thalamus most- ly with bilateral damage at any level of this system and interrupted pathways connecting the two hemispheres. At least partial cognitive function can be recovered following restricted bilateral injuries to the paramedian thalamus and mesencephalon $[28,29]$. In our study, we observed that patients with isolated lesions restricted to the bilateral paramedian thalamus and mesencephalon recovered to the state of MCS after being in an eyes-closed coma, in 3 months.

The study had some limitations. First, the follow-up period was relatively short. Our findings indicate that like PVS, MCS presented in one of seven patients as a transitional state arising during recovery from coma after acute stroke or after worsening of progressive stroke in one of 10 patients. A few studies have examined differences in outcome between PVS and MCS [30]. Studies examined either traumatic or nontraumatic injuries, and patients with MCS had significantly better outcomes compared with patients with PVS at 1 year [31]. Long-term outcome and transition between them after stroke warrant further investigation that are beyond the scope of the present study. Second, the 
cross-sectional design prevented us from investigating the relationship between neuroimaging markers and long-term outcome of consciousness states. However, in patients with stroke who remained in PVS at 3 months, neuroimaging findings showed that structural injuries within the corpus callosum and cingulate gyrus significantly predicted loss of self or environmental awareness as in previous studies [32,33]. Patients with isolated rostrocaudal midbrain and dorsolateral thalamic lesions showed transition from coma to MCS with signs of awareness, but those with additional supratentorial lesions did not recover and continued to live with PVS. The pathophysiological role of these structures on awareness have not been fully elucidated; interruption of the brainstem-hemispheric awareness network is probably one of the main contributors. Moreover, recent studies with functional MRI reported that patients with MCS showed more widespread activations with cortico-cortical functional connectivity compared with patients with PVS [34].

Our study had several strengths. First, the large sample size allowed us to define different aspects of two different consciousness levels and feed many factors into the regression model. Our study provides an original and reliable approach using OCQ in a large population with severe stroke and radiological examination to assess involved brain structures and relationship of poststroke consciousness states. Second, we analyzed most of the likely radiological determinants of poststroke consciousness states and delineated higher frequency of multiple lesions, WMH burden, and bilateral hemispheric lesions either in the anterior and posterior circulation, but these factors were not statistically different in both groups. Third, the present study clearly showed significant difference of awareness between PVS and MCS using OCQ to explore gestural or verbal responses, intelligible verbalization, and purposeful and affective behaviors. Fourth, we found higher frequency of involvement of strategic regions (i.e., left and right middle frontal gyrus, left and right middle and superior temporal gyrus, left and right parietal cortex [angular and supramarginal gyri], putamen, pallidum, cingulate gyrus and corpus callosum, anterior and middle parts of the left and right thalami, ventral and dorsal midbrain, and dorsal pons) in both groups and cingulate gyrus and corpus callosum involvement, which might be an independent risk factor for PVS [35,36]. A large body of evidence suggests that lesions in strategic areas have a key role in the development of consciousness disorders regardless of their volume but the associations between strategic sites and consciousness states warrant further investigation that are beyond the scope of our study. Fifth, we preferred to study both cerebral infarcts, hemorrhage, and all causes of stroke (large and small infarcts and all hemorrhages) so that our study cohort was repre- sentative of clinical populations. Furthermore, this design has the advantage of reducing multiple linearity and confirming stroke-related factors for all subtypes. Sixth, in our large cohort, we found a higher rate of previous neurocognitive disorder especially in patients with PVS and MCS, which might increase the risk of cognitive and awareness impairment [37,38]. Lastly, the present study was characterized by older age and higher stroke severity compared with population-based stroke studies [39]. The absence of patients with $\mathrm{mRS}$ score $<5$ is explained by the fact that patients with severe consciousness impairment were followed and their consciousness states were assessed in the stroke unit for 3 months. Furthermore, the present study was designed to focus on consciousness state of patients with mRS score 5 .

Finally, PVS and MCS are different consciousness states especially in terms of awareness function according to clinical and radiological findings. Cognitive capacities and performance of these patients should be well questioned after stroke to improve the determination of outcome of consciousness states. Further long-term clinical trials are warranted to determine the consciousness prognosis and late recovery of patients with PVS and MCS after stroke.

\section{ARTICLE INFORMATION}

\section{Conflict of interest}

No potential conflict of interest relevant to this article.

\section{Author contributions}

Conceptualization: EK and FEB. Data curation \& Formal analysis: All authors. Visualization \& Writing-original draft: All authors. Writing-review editing \& Supervision: EK and CEE.

\section{References}

1. Edelman GM, Tononi GA. A universe of consciousness: how matter becomes imagination. New York (NY): Basic Books; 2000.

2. Plum F. Coma and related global disturbances of the human conscious state. In: Peters A, Jones EG, editors. Cerebral cortex. Vol. 9. Boston (MA): Springer; 1991.p. 359-425.

3. Posner JB, Saper CB, Schiff ND, Plum F. Approach to management of the unconscious patient. In: Posner JB, Saper CB, Schiff ND, Plum F, editors. Plum and Posner's diagnosis of stupor and coma. 4th ed. London (UK): Oxford University Press; 2007. p. 309-30.

4. Jennett B, Plum F. Persistent vegetative state after brain damage. A syndrome in search of a name. Lancet 1972;1:734-7. 
5. Giacino JT, Ashwal S, Childs N, Cranford R, Jennett B, Katz DI, et al. The minimally conscious state: definition and diagnostic criteria. Neurology 2002;58:349-53.

6. Schiemanck SK, Kwakkel G, Post MW, Prevo AJ. Predictive value of ischemic lesion volume assessed with magnetic resonance imaging for neurological deficits and functional outcome poststroke: a critical review of the literature. Neurorehabil Neural Repair 2006;20:492-502.

7. Heldner MR, Zubler C, Mattle HP, Schroth G, Weck A, Mono ML, et al. National Institutes of Health Stroke Scale score and vessel occlusion in 2152 patients with acute ischemic stroke. Stroke 2013;44:1153-7.

8. Poisson SN, Nguyen-Huynh MN, Johnston SC, Furie KL, Lev $\mathrm{MH}, \mathrm{Smith}$ WS. Intracranial large vessel occlusion as a predictor of decline in functional status after transient ischemic attack. Stroke 2011;42:44-7.

9. Lövblad KO, Baird AE, Schlaug G, Benfield A, Siewert B, Voetsch B, et al. Ischemic lesion volumes in acute stroke by diffusion-weighted magnetic resonance imaging correlate with clinical outcome. Ann Neurol 1997;42:164-70.

10. Johnston KC, Connors AF Jr, Wagner DP, Knaus WA, Wang X, Haley EC Jr. A predictive risk model for outcomes of ischemic stroke. Stroke 2000;31:448-55.

11. Mijajlović MD, Pavlović A, Brainin M, Heiss WD, Quinn TJ, Ihle-Hansen HB, et al. Post-stroke dementia: a comprehensive review. BMC Med 2017;15:11.

12. Kumral E, Ozkaya B, Sagduyu A, Sirin H, Vardarli E, Pehlivan M. The Ege Stroke Registry: a hospital-based study in the Aegean region, Izmir, Turkey. Analysis of 2,000 stroke patients. Cerebrovasc Dis 1998;8:278-88.

13. Wilson JT, Hareendran A, Grant M, Baird T, Schulz UG, Muir $\mathrm{KW}$, et al. Improving the assessment of outcomes in stroke: use of a structured interview to assign grades on the modified Rankin Scale. Stroke 2002;33:2243-6.

14. Goldstein LB, Bertels C, Davis JN. Interrater reliability of the NIH stroke scale. Arch Neurol 1989;46:660-2.

15. Teasdale G, Knill-Jones R, van der Sande J. Observer variability in assessing impaired consciousness and coma.J Neurol Neurosurg Psychiatry 1978;41:603-10.

16. Adams HP Jr, Davis PH, Leira EC, Chang KC, Bendixen BH, Clarke WR, et al. Baseline NIH Stroke Scale score strongly predicts outcome after stroke: a report of the Trial of Org 10172 in Acute Stroke Treatment (TOAST). Neurology 1999;53:12631.

17. Multi-Society Task Force on PVS. Medical aspects of the persistent vegetative state (1). N Engl J Med 1994;330:1499-508.

18. Multi-Society Task Force on PVS. Medical aspects of the per- sistent vegetative state (2). N Engl J Med 1994;330:1572-9.

19. Wardlaw JM, Smith EE, Biessels GJ, Cordonnier C, Fazekas F, Frayne R, et al. Neuroimaging standards for research into small vessel disease and its contribution to ageing and neurodegeneration. Lancet Neurol 2013;12:822-38.

20. Fazekas F, Niederkorn K, Schmidt R, Offenbacher H, Horner S, Bertha $\mathrm{G}$, et al. White matter signal abnormalities in normal individuals: correlation with carotid ultrasonography, cerebral blood flow measurements, and cerebrovascular risk factors. Stroke 1988;19:1285-8.

21. Cordonnier C, Potter GM, Jackson CA, Doubal F, Keir S, Sudlow $\mathrm{CL}$, et al. Improving interrater agreement about brain microbleeds: development of the Brain Observer MicroBleed Scale (BOMBS). Stroke 2009;40:94-9.

22. Schnakers C, Vanhaudenhuyse A, Giacino J, Ventura M, Boly M, Majerus S, et al. Diagnostic accuracy of the vegetative and minimally conscious state: clinical consensus versus standardized neurobehavioral assessment. BMC Neurol 2009;9:35.

23. Childs NL, Mercer WN. Brief report: late improvement in consciousness after post-traumatic vegetative state. $\mathrm{N}$ Engl J Med 1996;334:24-5.

24. Kampfl A, Schmutzhard E, Franz G, Pfausler B, Haring HP, UImer $\mathrm{H}$, et al. Prediction of recovery from post-traumatic vegetative state with cerebral magnetic-resonance imaging. Lancet 1998;351:1763-7.

25. Laureys S, Owen AM, Schiff ND. Brain function in coma, vegetative state, and related disorders. Lancet Neurol 2004;3:53746.

26. Thomalla G, Hartmann F, Juettler E, Singer OC, Lehnhardt FG, Köhrmann M, et al. Prediction of malignant middle cerebral artery infarction by magnetic resonance imaging within 6 hours of symptom onset: a prospective multicenter observational study. Ann Neurol 2010;68:435-45.

27. Mega MS, Cohenour RC. Akinetic mutism: disconnection of frontal-subcortical circuits. Neuropsychiatry Neuropsychol Behav Neurol 1997;10:254-9.

28. Katz DI, Alexander MP, Mandell AM. Dementia following strokes in the mesencephalon and diencephalon. Arch Neurol 1987;44:1127-33.

29. van Domburg PH, ten Donkelaar HJ, Notermans SL. Akinetic mutism with bithalamic infarction. Neurophysiological correlates.J Neurol Sci 1996;139:58-65.

30. Giacino JT, Kalmar K. Diagnostic and prognostic guidelines for the vegetative and minimally conscious states. Neuropsychol Rehabil 2005;15:166-74.

31. Wei JW, Heeley EL, Wang JG, Huang Y, Wong LK, Li Z, et al. Comparison of recovery patterns and prognostic indicators for 
ischemic and hemorrhagic stroke in China: the ChinaQUEST (QUality Evaluation of Stroke Care and Treatment) Registry study. Stroke 2010;41:1877-83.

32. Németh G, Hegedüs K, Molnár L. Akinetic mutism associated with bicingular lesions: clinicopathological and functional anatomical correlates. Eur Arch Psychiatry Neurol Sci 1988;237:21822.

33. Qin P, Di H, Liu Y, Yu S, Gong Q, Duncan N, et al. Anterior cingulate activity and the self in disorders of consciousness. Hum Brain Mapp 2010;31:1993-2002.

34. Laureys S, Schiff ND. Coma and consciousness: paradigms (re) framed by neuroimaging. Neuroimage 2012;61:478-91.

35. Gosseries O, Di H, Laureys S, Boly M. Measuring consciousness in severely damaged brains. Annu Rev Neurosci 2014;37:457-
78.

36. Giacino JT, Fins JJ, Laureys S, Schiff ND. Disorders of consciousness after acquired brain injury: the state of the science. Nat Rev Neurol 2014;10:99-114.

37. Go RC, Todorov AB, Elston RC, Constantinidis J. The malignancy of dementias. Ann Neurol 1978;3:559-61.

38. Barba R, Morin MD, Cemillán C, Delgado C, Domingo J, Del Ser T. Previous and incident dementia as risk factors for mortality in stroke patients. Stroke 2002;33:1993-8.

39. Béjot Y, Rouaud O, Jacquin A, Osseby GV, Durier J, Manckoundia $\mathrm{P}$, et al. Stroke in the very old: incidence, risk factors, clinical features, outcomes and access to resources: a 22-year population-based study. Cerebrovasc Dis 2010;29:111-21. 